\title{
PAPEL DO EXERCÍCIO FÍSICO E DA NUTRIÇÃO PARA A PREVENÇÃO E TRATAMENTO DA OSTEOPOROSE*
}

\author{
Jonas Silva de Carvalho ${ }^{1}$ \\ Lorena Cristina Curado Lopes ${ }^{2}$ \\ João Felipe Mota ${ }^{3}$
}

\section{Introdução}

A osteoporose é definida como desordem esquelética caracterizada por comprometimento da qualidade (estrutura óssea) e da quantidade óssea (densidade) que predispõe ao aumento do risco de fratura (CONSENSO NHI, 2000). Dentre as fraturas induzidas pela osteoporose, aquelas que ocorrem na articulação do quadril e na coluna vertebral são as mais graves, pois resultam em hospitalização e com frequência estão relacionadas com incapacidade ou morte. Estas fraturas e internações relacionadas com a doença, possuem um elevado custo. No Brasil, estima-se que 310 milhões de dólares por ano são gastos com esta doença (AZIZIYEH et al., 2019).

Tendo em vista o aumento da prevalência da osteoporose, devido ao envelhecimento populacional (BOSCHITSCH; DURCHSCHLAG; DIMAI, 2017). Torna-se de suma importância que os profissionais da saúde compreendam e incentivem um estilo de vida saudável tanto para prevenção quanto para o tratamento da osteoporose. As estratégias preventivas devem incluir um programa de exercício físico supervisionado prescrito conforme as necessidades e especificidades deste público, bem como uma alimentação equilibrada com nutrientes que influenciam positivamente a saúde óssea.

Desta forma esta revisão narrativa de literatura tem como objetivo sumarizar as evidências científicas sobre o efeito da dieta e do exercício físico na prevenção e tratamento da osteoporose.

\footnotetext{
* 10.29388/978-65-86678-46-8-0-f.145-156

${ }^{1}$ Graduado em Educação Física pela UNIFIMES.

${ }^{2}$ Docente efetiva da UNIFIMES. Doutoranda em Ciências da Saúde pela UFG.

${ }^{3}$ Professor do Programa de Pós-Graduação em Ciências da Saúde (UFG).
} 


\section{Nutrição no manejo da osteoporose}

A nutrição adequada desempenha um papel importante na prevenção e tratamento da osteoporose (NIEVES, 2005). O cálcio e a vitamina D são os nutrientes de maior importância, todavia uma dieta equilibrada e variada é essencial na prevenção de perda e fratura óssea (NIEVES, 2005; TUCKER, 2009). A ingestão inadequada de cálcio e vitamina D influencia nas concentrações dos hormônios reguladores do cálcio (NIEVES, 2005). A deficiência desses nutrientes resulta em menor absorção de cálcio e menor concentração de cálcio ionizado circulante, estimulando a secreção do hormônio da paratireoide (PTH). O efeito cumulativo de elevadas concentrações de PTH (hiperparatireoidismo secundário) é o aumento na remodelação óssea, com perda significativa de osso e maior risco de fratura (NIEVES, 2005).

No Brasil, estima-se que a ingestão diária de cálcio esteja abaixo das recomendações ( 400mg) (PINHEIRO et al., 2009) e que a prevalência de deficiência e insuficiência de vitamina $\mathrm{D}$ sejam respectivamente de $28,2 \%$ e 45,3\% (PEREIRA-SANTOS et al., 2019). A quantidade ótima de diária de cálcio e vitamina $\mathrm{D}$ variam de acordo com o sexo e faixa etária e são apresentadas na tabela 1 .

A suplementação de cálcio ainda é controversa devido aos inúmeros estudos que a associam a suplementação com cálculos renais (COE; WORCESTER; EVAN, 2016) e doenças cardiovasculares (ANDERSON et al., 2016). Um dos pontos conflitantes é que a maioria dos estudos associam vitamina $\mathrm{D}$ a suplementação de cálcio, comprometendo o entendimento dos efeitos isolados destes nutrientes na redução da perda óssea.

Dessa forma, a maior ingestão de alimentos fontes em cálcio e vitamina $\mathrm{D}$ é recomendada como estratégia primária. O cálcio pode ser encontrado principalmente em laticínios, vegetais verde escuros, peixes enlatados, crustáceos, nozes e castanhas, bem como alimentos fortificados (NIEVES, 2005). Uma lista com alimentos fonte em cálcio pode ser observada na tabela 2. Vale ressaltar que a presença de ácidos fítico e oxálico reduzem a biodisponibilidade do cálcio e são encontrados em farelos de cereais, grãos secos e vegetais. Logo, as melhores fontes de cálcio ainda são as provenientes de origem animal, as quais apresentam maior taxa de absorção do nutriente (INTERNATIONAL LIFE SCIENCES, 2018) . Caso não seja possível uma ingestão adequada de cálcio por meio da dieta, sua suplementação pode ser necessária, mas não deve exceder 
500 a $600 \mathrm{mg}$ por dose, visto que o fracionamento aumenta a absorção (WEAVER, CONNIE M., HEANEY, 2006). A vitamina D pode ser encontrada em alimentos (tabela 3), mas estima-se que em humanos 80 a 90\% seja adquirida pela síntese cutânea a partir da radiação solar ultravioleta (INTERNATIONAL LIFE SCIENCES INSTITUTE, 2018).

As necessidades nutricionais para otimizar a saúde óssea podem ser facilmente atendidas por uma dieta equilibrada composta por frutas, legumes, laticínios e outros alimentos proteicos, mas restrita em alimentos com baixa densidade de nutrientes e ricos em sódio, que aumenta a excreção renal do cálcio. Alimentos são preferidos para manter o equilíbrio de cálcio, até porque outros nutrientes essenciais são encontrados em alimentos fontes em cálcio. Outros nutrientes importantes para manutenção da saúde óssea são o magnésio e potássio, os quais ajudam a manter o equilíbrio ácido-base, a vitamina $\mathrm{C}, \mathrm{K}$ e complexo $\mathrm{B}$, bem como os carotenoides e outros compostos que contribuem com a defesa antioxidante (NIEVES, 2005; TUCKER, 2009). Para adequar o consumo desses nutrientes e compostos, a ingestão variada de frutas e vegetais torna-se importante. As proteínas também beneficiam a manutenção do tecido ósseo e sua baixa ingestão tem sido associada ao aumento do risco de fraturas em idosos (TUCKER, 2009).

Tabela 1. Recomendações nutricionais (RDA) para cálcio e vitamina D de acordo com o estágio de vida (quantidade / dia).

\begin{tabular}{cccc}
\hline \multirow{2}{*}{ Idade (anos) } & \multicolumn{2}{c}{ RDA Cálcio (mg) } & RDA Vitamina D (IU/ Og) \\
\cline { 2 - 4 } & Mulheres & Homens & Mulheres e Homens \\
\hline $4-8$ & 1000 & 1000 & $600 / 15$ \\
$9-18$ & 1300 & 1300 & $600 / 15$ \\
$19-50$ & 1000 & 1000 & $600 / 15$ \\
$51-70$ & 1200 & 1000 & $600 / 15$ \\
$>70$ & 1200 & 1200 & $800 / 20$ \\
\hline
\end{tabular}

Tabela 2. Alimentos fontes em cálcio.

\begin{tabular}{lccc}
\hline \multicolumn{1}{c}{ Alimento } & Porção & Quantidade (g) & Cálcio (mg) \\
\hline Camarão 7 barbas, frito & 5 unidades & 150 & 1.440 \\
Pescada frita & 1 filé médio & 120 & 454 \\
Sardinha em lata & 3 unidades & 72 & 396 \\
Leite fortificado com cálcio* & 1 copo & 240 & 384
\end{tabular}




\begin{tabular}{lccc} 
Queijo Fresco & 2 fatias médias & $60 \mathrm{~g}$ & 347 \\
Leite Desnatado & 1 copo & 240 & 322 \\
Leite Integral & 1 copo & 240 & 295 \\
Iogurte Natural & 1 potinho & 200 & 286 \\
Queijo Mussarela & 2 fatias finas & 30 & 262 \\
Espinafre Cozido & 1 xícara & 190 & 213 \\
Couve refogada & 5 colheres de sopa & 100 & 177 \\
Ricota & 2 fatias médias & 60 & 152 \\
Iogurte de morango & 1 potinho & 130 & 130 \\
\hline
\end{tabular}

Adaptado de: (TACO, 2011) * Informação do fabricante.

Tabela 3. Alimentos fontes em vitamina D.

\begin{tabular}{|c|c|c|c|}
\hline Alimento & Porção & Quantidade (g) & $\begin{array}{c}\text { Vitamina D } \\
\text { (UI) }\end{array}$ \\
\hline Óleo de fígado de peixe & 1 colher de sopa & 0,23 & 924 \\
\hline Cavalinha grelhada & 1 filé médio & 100 & 352 \\
\hline Salmão grelhado & 1 filé médio & 120 & 341 \\
\hline Atum enlatado em salmoura & 1 lata drenada & 120 & 173 \\
\hline Cereal matinal fortificado & 3/4 xícara & $30 \mathrm{~g}$ & 156 \\
\hline Sardinha enlatada em salmoura & 1 lata drenada & 84 & 155 \\
\hline Ovo de galinha cozido & 1 unidade média & 50 & 44 \\
\hline Fígado de boi frito & 1 unidade grande & 130 & 47 \\
\hline Margarina fortificada & 1 colher de sopa & 10 & 31 \\
\hline
\end{tabular}

Adaptado: International Osteoporosis Foundation (2006)

\section{Exercícios para a prevenção da osteoporose}

O exercício físico é amplamente aceito como estratégia efetiva para a melhoria e manutenção da saúde óssea em todas as idades. A prática de exercício físico especialmente durante a fase da adolescência tem sido considerada um "janela de oportunidade" para maximizar a saúde óssea (GOING, SCOTT B; FARR, JOSHUA, 2010). Durante esta fase, quando submetido a tensão, o tecido ósseo aumentaria a atividade osteogênica, resultando em maior densidade mineral óssea, que sustentada ao longo da vida auxilia na prevenção da osteoporose. Por este motivo, durante esta fase da vida, atividades de impacto, como saltos corridas e esportes de contato devem ser estimuladas (TROY et al., 2018). A figura 1 representa a dinâmica da formação óssea ao longo da vida, mostrando que o pico de massa óssea ocorre aproximadamente aos 18 anos e 
continua crescendo de forma mais sutil até os 30 anos, e a no período da menopausa existe uma redução acentuada da densidade óssea.

Figura 1: Pico de o índice do pico de massa óssea atingido durante a vida.

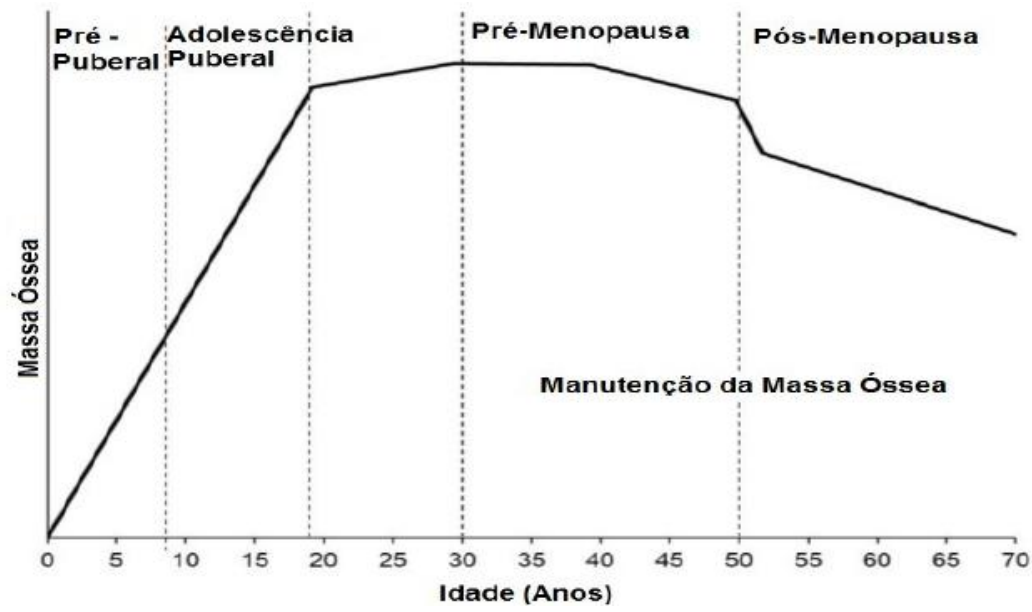

Fonte: Adaptado de Troy et al (2018).

De fato, vários estudos mostram que atletas engajados em exercícios de impacto, como exemplo ginástica, basquete, voleibol, levantamento de peso, possuem densidade mineral óssea superior quando comparados aos pares não atletas ou atletas de esporte de baixo impacto como o caso da natação e do ciclismo (ABRAHIN et al., 2016). Contudo, as evidências atuais indicam que os efeitos do exercício osso são dependentes da modalidade, dose e intensidade. Do ponto de vista fisiológico, é a tensão e a deformação a que osso é submetido em resposta a uma carga mecânica, imposta durante o exercício físico que leva uma resposta osteogênica (AIRES, 2017). Por isso, nem todas as modalidades esportivas possuem o mesmo efeito osteogênico.

De acordo com a Sociedade Australiana de Medicina Esportiva (BECK et al., 2017), o programa de prevenção da osteoporose em indivíduos com baixo ou moderado risco sem fatores de risco para quedas, devem incluir no programa de treinamento:

- Exercícios de saltos verticais e multidirecionais, (50 saltos por sessão, divididos entre 3-5 séries, de 10 a 20 repetições com intervalo entre 1-2 minutos e frequência entre 4 e 7 vezes por semana); 
- Treinamento resistido com foco em exercícios multiarticulares para os maiores grupos musculares e intensidade $85 \%$ de uma repetição máxima (RM);

- Exercícios de equilíbrio: e exercícios que desafiem a estabilidade, todas estas recomendações devem ser seguidas conforme tolerância individual.

\section{Prescrição de exercício para indivíduos com osteoporose}

Embora a prevenção da osteoporose se inicie precocemente. Nunca é tarde para iniciar um programa de exercícios a fim de melhorar a saúde óssea. Todo programa de treinamento desenvolvido para prevenir fraturas, particularmente aqueles com fatores de risco conhecidos para queda, deve incluir atividades para otimizar a função muscular, o equilíbrio e a estabilidade da marcha (BECK et al., 2017; GIANGREGORIO et al., 2014).

Em relação a dose de exercício necessária para prevenir quedas foi mostrado que no mínimo 2,5 sessões de exercício semanais são necessárias em idosos (KEMMLER; VON STENGEL; KOHL, 2017). Em concordância, em indivíduos com osteoporose, a dose mínima de exercício parece ser similar. Borba Pinheiro et al. (2016) testaram o efeito do treinamento resistido realizado duas ou três vezes por semana sobre a densidade mineral óssea, força e capacidade funcional em mulheres com osteoporose. O programa de treino teve 13 meses de duração e intensidade progressivamente aumentada (60 a $90 \%$ de $1 \mathrm{RM}$ ), as mulheres que treinaram 3 vezes na semana obtiveram os maiores ganhos da densidade mineral óssea, força muscular e capacidade funcional quando comparados as mulheres que treinaram duas vezes por semana. Contudo, o treino realizado duas vezes por semana teve maior efeito do que o grupo que não treinou. Estes dados sugerem que o efeito protetor do treinamento é dose dependente e que maiores doses possuem maior efeito na prevenção de quedas.

Ainda sobre o ponto de vista de variáveis de prescrição de treinamento, as recomendações vigentes sugerem que os maiores ganhos de DMO são advindos de programas de treinamento que utilizam intensidades mais altas $(80-85 \% \mathrm{RM})$. Contudo, é importante ressaltar, que para aqueles indivíduos previamente sedentários o treinamento deve iniciar em intensidades mais baixas e progressivamente alcançar as intensidades mais altas (GIANGREGORIO et al., 2014). 
O estudo de Watson et al (2018) corroboram estas recomendações. Os autores avaliaram o efeito de 8 meses de treinamento resistido em 92 mulheres com osteopenia ou osteoporose. As sessões de exercício foram realizadas duas vezes por semana durante 30 minutos (5 séries de 5 repetições com carga entre 80 e $85 \%$ de $1 \mathrm{RM}$ ) e compreenderam os seguintes exercícios: levantamento terra, desenvolvimento e agachamento, combinados com um programa de exercícios com saltos. Os indivíduos que realizaram este treinamento, considerado de alta intensidade, obtiveram maiores ganhos na capacidade funcional e densidade mineral óssea, especialmente no colo de fêmur, com amento de 9,8\% após o período de treinamento.

Em relação ao treinamento aeróbio, como a caminhada (frequentemente indicada a estes pacientes), quando realizado de forma isolada exerce estímulo insuficiente para o aumento da DMO, ou mesmo prevenção de quedas (GIANGREGORIO et al., 2014). Contudo, quando combinado com o treinamento resistido (tipo de treino conhecido como treino concorrente) parece aumentar a magnitude do ganho da densidade mineral óssea. Uma meta-análise avaliou o impacto de programas de exercício físico sobre a densidade mineral óssea em mulheres no período da pós-menopausa, e mostrou que o treinamento concorrente tem maior efeito sobre o colo do fêmur e vértebras lombares do que o treinamento resistido isolado (ZHAO, XU, 2015). Assim, o treinamento aeróbio, como caminhada pode ser incluído no programa de treinamento, e deve ser uma estratégia preferencialmente adotada nos indivíduos com fraturas vertebrais ou outras comorbidades que limitam a realização de exercícios de maior impacto (GIANGREGORIO et al., 2014).

Um estudo realizado por Arazi e colaboradores (2018) testou o efeito do treinamento concorrente associado ao consumo de leite em mulheres com diagnóstico de osteoporose. Neste estudo, as voluntárias foram randomizadas em 4 grupos de intervenção com duração de 10 semanas: G1: realizou treinamento concorrente juntamente com consumo de leite (aeróbico 20-45 min entre 55 a 75\% da frequência cardíaca máxi$\mathrm{ma}+$ exercícios resistidos a $80 \%$ de $1 \mathrm{RM}$ ) e estes consumiam $500 \mathrm{ml}$ de leite ( $250 \mathrm{ml}$ antes do treino e $250 \mathrm{ml}$ uma hora após o treino); G2 realizou apenas o treinamento concorrente igual a $G 1 ; G 3$, consumiu apenas leite $(500 \mathrm{ml})$ e o grupo controle não consumiu leite nem realizou treinamento. Os autores identificaram que o treinamento concorrente combinado com o consumo de leite, fonte em cálcio, teve maior impacto sobre 
a densidade mineral óssea das mulheres, seguido do grupo que realizou apenas treinamento concorrente.

Exercícios que desafiem o equilíbrio tais como exercícios com redução gradual da base de apoio, atividades que desafiem o deslocamento tradicional com mudanças de direção, inclinação, manutenção de posturas com os olhos vendados, são recomendados para melhoria do equilíbrio. A indicação é de que cada exercício deva ser realizado por no mínimo 10 segundos, 30 minutos por dia 4 vezes por semana (BECK et al., 2017). Estes exercícios podem ser feitos em pequenos blocos ao longo do dia, ou incorporados dentro da sessão de exercício (GIANGREGORIO et al., 2014).

Em relação a escolha dos exercícios, o profissional de educação física deve evitar prescrever movimentos de flexão e rotação de coluna. Um estudo clássico realizado com 59 pacientes com diagnóstico de osteoporose com idade 49 a 60 anos, observou que 89\% dos indivíduos com osteoporose que realizaram programa de treinamento com flexão de tronco, tiveram compressão vertebral. Estes dados sugerem que indivíduos com osteoporose possuem maior suscetibilidade de lesão na coluna, por isso deve-se ter uma atenção redobrada, evitando prescrição de atividades, especialmente movimentos de flexão e rotação de tronco (SINAKI; MIKKELSEN; BETH, 1984).

Outro cuidado importante que o profissional deve ter ao implementar o programa de exercícios é reduzir os riscos de quedas durante a execução do movimento. Dessa forma, desnivelamentos no piso, superfícies escorregadias devem ser atentamente detectadas e corrigidas. Adicionalmente, o profissional deve avaliar os riscos e benefícios dos exercícios para cada sujeito.

\section{Conclusão}

O exercício físico associado a dieta adequada são estratégias não farmacológicas fundamentais tanto para a prevenção da osteoporose, quanto para prevenção de quedas, fraturas e suas complicações em indivíduos com osteoporose. Evidências científicas aqui mostradas, indicam que os exercícios de maior impacto como corridas e saltos possuem maior efeito sobre a densidade mineral óssea, contudo, em indivíduos com alto risco de fratura na coluna vertebral, os riscos associados a este tipo de exercício podem ser maiores do que os benefícios. Além disso, o exercício quando associado a uma dieta equilibrada fonte em nutrientes 
envolvidos com o metabolismo ósseo apresenta maiores efeitos sobre a saúde óssea. Portanto, torna-se imprescindível a avaliação profissional individualizada para a correta prescrição.

\section{Referências}

ABRAHIN, O. et al. Natação e ciclismo não causam efeitos positivos na densidade mineral óssea: uma revisão sistemática. Revista Brasileira de Reumatologia, v. 56, n. 4, p. 345-351, 2016.

ANDERSON, J. J. B. et al. Calcium intake from diet and supplements and the risk of coronary artery calcification and its progression among older adults: 10year follow-up of the multi-ethnic study of atherosclerosis (MESA). Journal of the American Heart Association, v. 5, n. 10, p. 1-13, 2016.

ARAZI, H.; SAMADPOUR, M.; EGHBALI, E. The effects of concurrent training (aerobic-resistance) and milk consumption on some markers of bone mineral density in women with osteoporosis. BMC Women's Health, v. 18, n. 1, 2018.

AZIZIYEH, R. et al. The burden of osteoporosis in four Latin American countries: Brazil, Mexico, Colombia, and Argentina. Journal of Medical Economics, v. 22, n. 7, p. 638-644, 2019.

BECK, B. R. et al. Exercise and Sports Science Australia (ESSA) position statement on exercise prescription for the prevention and management of osteoporosis. Journal of Science and Medicine in Sport, v. 20, n. 5, p. 438-445, 2017.

BOLLAND, M. J. et al. Calcium supplements with or without vitamin D and risk of cardiovascular events: Reanalysis of the Women's Health Initiative limited access dataset and meta-analysis. Bmj, v. 342, n. 7804, 2011.

BORBA-PINHEIRO, C. J. et al. Resistance training programs on bone related variables and functional independence of postmenopausal women in pharmacological treatment: A randomized controlled trial. Archives of Gerontology and Geriatrics, v. 65, n. 40, p. 36-44, 2016.

BOSCHITSCH, E. P.; DURCHSCHLAG, E.; DIMAI, H. P. Age-related prevalence of osteoporosis and fragility fractures: real-world data from an Austrian Menopause and Osteoporosis Clinic. Climacteric, v. 20, n. 2, p. 157-163, 2017. 
COE, F. L.; WORCESTER, E. M.; EVAN, A. P. Idiopathic hypercalciuria and formation of calcium renal stones. Nature Reviews Nephrology, v. 12, n. 9, p. 519-533, 2016.

CONFERENCE, C. Osteoporosis prevention, diagnosis, and therapy. NIH consensus statement, v. 17, n. 1, p. 1-45, 2000.

FOUNDATION, N. O. Bone Appétit The role of food and nutrition in building. International Osteoporosis Foundation, 2006.

GIANGREGORIO, L. M. et al. Too Fit to Fracture: Exercise recommendations for individuals with osteoporosis or osteoporotic vertebral fracture. Osteoporosis International, v. 25, n. 3, p. 821-835, 2014.

GOING, SCOTT B; FARR, JOSHUA, N. of Opportunity for Osteoporosis Prevention? Int J Compos Res, v. 8, p. 1-9, 2010.

INTERNATIONAL LIFE SCIENCES INSTITUTE DO BRASIL. Calcio. In: Journal of Chemical Information and Modeling. v. 1, p. 1689-1699.

KEMMLER, W.; VON STENGEL, S.; KOHL, M. Exercise Frequency and Fracture Risk in Older Adults-How Often Is Enough? Current Osteoporosis Reports, v. 15, n. 6, p. 564-570, 2017.

NIEVES, J. W. Osteoporosis: The role of micronutrients. American Journal of Clinical Nutrition, v. 81, n. 5, p. 1232-1239, 2005.

PEDERSEN, B. K.; SALTIN, B. Exercise as medicine - Evidence for prescribing exercise as therapy in 26 different chronic diseases. Scandinavian Journal of Medicine and Science in Sports, v. 25, p. 1-72, 2015.

PEREIRA-SANTOS, M. et al. Epidemiology of vitamin D insufficiency and deficiency in a population in a sunny country: Geospatial meta-analysis in Brazil. Critical Reviews in Food Science and Nutrition, v. 59, n. 13, p. 2102-2109, 2019.

PINHEIRO, M. M. et al. Nutrient intakes related to osteoporotic fractures in men and women - The Brazilian Osteoporosis Study (BRAZOS). Nutrition Journal, v. 8, n. 1, p. 1-8, 2009.

SINAKI, M.; MIKKELSEN, B. A.; BETH, A. Postmenopausal spinal osteoporosis: Flexion versus extension exercises Archives of Physical Medicine and Rehabilitation, 1984.

TACO. Tabela brasileira de composição de alimentos NEPA - Unicamp, 2011. Disponível em: <http://www.unicamp.br/nepa/taco/>. 
TROY, K. L. et al. Exercise early and often: Effects of physical activity and exercise on women's bone health. International Journal of Environmental Research and Public Health, v. 15, n. 5, 2018.

TUCKER, K. Osteoporosis: nutrition. Epidemiology and Pathophysiology, v. Suppl, p. 131-135, 2009.

WATSON, S. L. et al. High-Intensity Resistance and Impact Training Improves Bone Mineral Density and Physical Function in Postmenopausal Women With Osteopenia and Osteoporosis: The LIFTMOR Randomized Controlled Trial. Journal of Bone and Mineral Research, v. 33, n. 2, p. 211-220, 2018.

WEAVER, CONNIE M., HEANEY, R. P. Calcium in Human Health. [s.l: s.n.]. 
